\title{
Recent Changes to the Balkan Medical Journal
}

There have been several new improvements to the Balkan Medical Journal to enhance its quality and importance. These include an updated and detailed "Instructions to Authors" section, an "Editorial Policy" section with information on the structure and duration of editorial workflow and the publication process, and a new "Instructions to Reviewers" section. We strongly recommend that our readers, authors, and reviewers refer to these sections for an idea of the evaluation and selection process for papers submitted to the Balkan Medical Journal.

In addition, the Editorial Board of the journal has expanded through acquiring distinguished colleagues from different medical specialisations and from different countries of the Balkans and all over the World. We are committed to building a supportive and vibrant community of scholars to take the journal forward.

Other important changes to the Balkan Medical Journal include the acquisition of the ScholarOne Manuscripts peer review system and PRS Proofreading and Copyediting Services. These two systems are used by many of the major journals. The journal's website is also about to be changed to a more user friendly-format with richer content.
Moreover, the Balkan Medical Journal is now a member of important organisations including the International Council of Medical Journal Editors (ICMJE), the World Association of Medical Editors (WAME), the Council of Science Editors (CSE), and the European Association of Science Editors (EASE), and is committed to following their instructions.

All of these changes are made in order to help our authors, reviewers, and editors to increase the quality of their work and to reduce the duration and burden of the evaluation and publication process. I thank the members of the Editorial Board and the Publisher, who are the primary forces behind the recent progress of the journal. I also thank Prof. Yener Yörük, Rector of Trakya University, and Prof. Hasan Sunar, Dean of the Faculty of Medicine, for their continuous support to the journal, and guaranteeing its editorial independence.

\author{
Professor Cem UZUN, MD \\ Editor-in-Chief, Balkan Medical Journal \\ Department of Otolaryngology, Trakya University \\ Faculty of Medicine, Edirne, Turkey
}

